\title{
Gene expression level of renalase in the skeletal muscles is increased with high-intensity exercise training in mice on a high-fat diet
}

\section{KATSUYUKI TOKINOYA ${ }^{1,2}$ (D), SEIKO ONO ${ }^{3}$, KAI AOKI ${ }^{2,4}$, KOKI YANAZAWA ${ }^{3}$, YASUHIRO SHISHIKURA ${ }^{3}$, TAKEHITO SUGASAWA ${ }^{4}$ and KAZUHIRO TAKEKOSHI ${ }^{4 *}$}

\footnotetext{
${ }^{1}$ Department of Health Promotion Sciences, Graduate School of Human Health Sciences, Tokyo Metropolitan University, Tokyo,192-0397, Japan

2 Japan Society for the Promotion of Science, Tokyo 102-0083, Japan

${ }^{3}$ Graduate School of Comprehensive Human Sciences, University of Tsukuba, Tsukuba 305-8577, Japan

${ }^{4}$ Division of Clinical Medicine, Faculty of Medicine, University of Tsukuba, Tsukuba 305-8577, Japan
}

Received: September 16, 2020 • Accepted: March 30, 2021

Published online: June 29, 2021

(C) 2021 Akadémiai Kiadó, Budapest

\begin{abstract}
Introduction: Exercise training is beneficial for reducing obesity. In particular, exercise training can lower the catecholamine concentration in circulation. Renalase, whose expression was first confirmed in the kidneys, is a physiologically active substance that decomposes circulating catecholamines; additionally, it has been reported to be present in the skeletal muscles. The aim of this study was to clarify the expression of renalase in the skeletal muscles and kidneys after high-intensity exercise training in obese mice. Material and methods: The mice were divided into four groups: normal diet and sedentary, normal diet and exercise training, highfat diet and sedentary, and high-fat diet and exercise training, and the test was performed for 8 weeks. Results: Body weight and skeletal muscle wet weight were reduced by high-fat diet intake but were rescued by training. Skeletal muscle renalase gene expression was significantly increased by exercise training. However, in the kidneys the gene expression of renalase was significantly increased by high-fat diet intake and exercise training. No significant changes were observed in the gene expression of catecholamine-degrading enzymes, catechol-O-methyltransferase and monoamine oxidase A and B. Conclusion: We demonstrated that exercise training increased the gene expression of renalase in the skeletal muscles and kidneys, thus lowering circulating catecholamine levels. This may lead to amelioration of obesity as catecholamines are lipolytic.
\end{abstract}

\footnotetext{
* Corresponding author. Division of Clinical Medicine, Faculty of Medicine, University of Tsukuba, 1-1-1 Tennodai, Tsukuba, Ibaraki 305-8577, Japan. Tel.: +81 29853 3389. E-mail: k-takemd@md.tsukuba.ac.jp
} 


\section{KEYWORDS}

blood pressure, catecholamine, kidney, obesity, skeletal muscle

\section{INTRODUCTION}

Obesity increases the risk of various diseases including diabetes, and exercise training is a tool to decrease this risk. In addition, diabetes induces catecholamine levels in the blood [1]. It has been reported that exercise training leads to an improvement in cardiovascular function, metabolism, and obesity $[2,3]$. Plasma levels of catecholamines, including adrenaline, noradrenaline, and dopamine, in previously sedentary subjects were decreased by exercise training, although these were increased by acute exercise [4,5]. Illi et al. [6] showed that plasma catecholamine levels did not change in sedentary subjects or those who exercised using inhibitors of catechol-O-methyltransferase (COMT) and/or monoamine oxidase A (MAO-A), which are catecholaminedegrading enzymes. Thus, the details of the metabolism of catecholamines remain unclear.

Renalase, a flavin adenine dinucleotide (FAD)-dependent soluble MAO, metabolizes catecholamines and regulates heart rate and blood pressure [7-9]. Its expression has been identified in other tissues, including skeletal muscles, cardiomyocytes, and intestines despite high expression in the kidneys [9]. In previous studies, renalase concentration in blood was shown to be increased in hypertensive patients and after exercise $[1,10,11]$. In addition, renalase knockout mice had higher catecholamine levels compared to wild-type mice [8]. Moreover, Tokinoya et al. [12] showed that renalase expression in the skeletal muscles and blood is increased by moderateintensity exercise. Thus, renalase concentration is increased by acute exercise, whereas it is unknown whether it is increased in obesity. Moreover, renalase mRNA content is increased by adrenaline in $\mathrm{C} 2 \mathrm{C} 12$ myotubes in vitro [13]. It has been reported that the expression of Sp1, ZBP89, and STAT3 are renalase regulators of adrenaline [14]. In addition, these transcriptional factors in the skeletal muscle were increased by acute exercise [12]. Furthermore, Tokinoya et al. [15] showed that renalase protein expression in the skeletal muscles is increased by low- and high-intensity exercise, despite decreased renalase expression in the kidneys [15]. Thus, renalase expression in the skeletal muscles is increased by acute exercise at various intensities.

However, it is unclear whether renalase expression in the skeletal muscles is increased by exercise training, although it is increased by acute exercise. In addition, the link between renalase expression in the skeletal muscles and obesity remains unclear. We hypothesize that renalase contributes to decreased circulating catecholamine levels in normal subjects with exercise training. This study aimed to elucidate the renalase level and factors related to metabolism of catecholamines in the skeletal muscles of mice with high-fat diet-induced obesity and exercise training.

\section{MATERIALS AND METHODS}

\section{Animals and experimental design}

All the experimental protocols used in the study are in accordance with the principles and guidelines of animal care of the Physiological Society of Japan and The Animal Ethics Committee of the University of Tsukuba (approval number: 19-121). 
Three-week-old male C57BL/6J mice (Japan SLC, Shizuoka, Japan) were used for the experiments. The mice were housed in a room at $20-26{ }^{\circ} \mathrm{C}$ with humidity of $40 \%-60 \%$ and a $12 \mathrm{~h}$ light-dark cycle. The study protocol is shown in Fig. 1. The mice were divided into four groups: normal diet and sedentary (ND-Sed), high-fat diet and sedentary (HFD-Sed), normal diet and high-intensity exercise training (ND-Tr), HFD and high-intensity exercise training (HFD-Tr). Animals were fed a normal chow diet (MF, Oriental Yeast, Tokyo, Japan) or high-fat diet (60\% fat; D12492, Research Diet, New Brunswick, USA) and were given water ad libitum. All mice were familiarized with a motor-driven horizontal treadmill (TMS-4B, MELQUEST, Toyama) for $30 \mathrm{~min}$ using mild electric shocks $(0.1 \mathrm{~mA})$ at the rear end of the treadmill and were put on a 3-day program after one week of preliminary breeding. Thereafter the mice were divided into sedentary or exercise training groups. Training was conducted for 5 days/week for 8 weeks. The mice trained at a speed of $25 \mathrm{~m} / \mathrm{min}$ for $30 \mathrm{~min}$, according to a high-intensity exercise protocol established by Okamoto et al. [16]. In this study, blood lactate was measured to confirm the exercise intensity in the training session of the last week. After the last training day, mice were fasted for $16 \mathrm{~h}$. The next day, glucose solution was administered intraperitoneally $(1.5 \mathrm{~g} / \mathrm{kg})$, and blood glucose levels were measured at 0-120 min. Then, samples from the gastrocnemius, soleus, plantaris muscles, kidneys, and white adipose tissue (WAT) were collected and stored at $-80{ }^{\circ} \mathrm{C}$ for subsequent analysis.

\section{Real-time quantitative reverse transcription PCR}

mRNA isolation was carried out using the following method. Tissues collected from the skeletal muscles, kidneys, heart, liver, and lungs were homogenized in a TissueLyser bead mixer (Qiagen, Hilden, Germany) at a frequency of $25 \mathrm{~Hz}$ for 2-5 min. Total mRNA was isolated using the Sepasol-RNA I Super G kit (Nacalai Tesque, Kyoto, Japan), according to the manufacturer's instructions. The total RNA concentration was measured at $260 \mathrm{~nm}$ using the NanoDrop 2000 Spectrophotometer (Thermo Fisher Scientific, MA, USA). The samples were then frozen and stored at $-80{ }^{\circ} \mathrm{C}$ for further analysis.

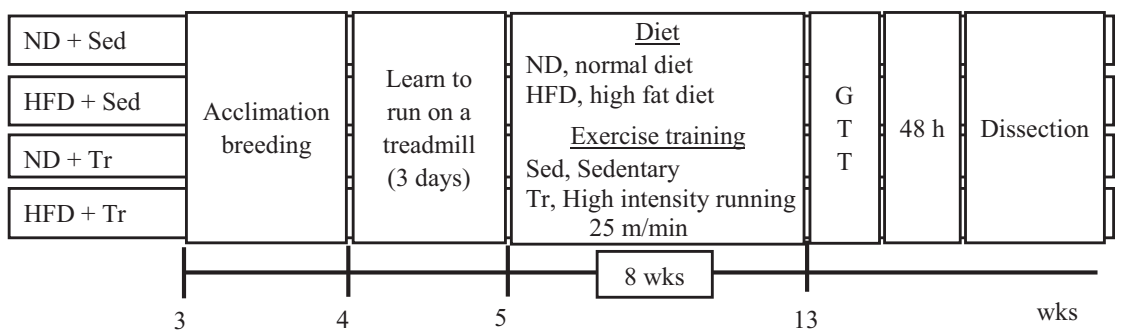

Fig. 1. Experimental protocol

The mice were divided into four groups: ND + Sed, HFD + Sed, ND + Tr, HFD + Tr before acclimation breeding. There was a rest for 2 days after learning to run for 3 days. The measurement of body weight was performed every week, and fresh feed was provided every third day. The mice were fasted for $16 \mathrm{~h}$ to perform the GTT after the final training session. The dissection was performed at $48 \mathrm{~h}$ after GTT. ND, normal diet;

HFD, high-fat diet; Sed, sedentary; Tr, high-intensity exercise training; GTT, glucose tolerance test 
Reverse transcription was performed using the following method. Total RNA was reverse transcribed into cDNA using PrimeScript RT Master Mix (Perfect Real Time; Takara Bio, Shiga, Japan). To quantify the gene expression levels, PCR was carried out using the KAPA SYBR FAST qPCR Master Mix kit (Kapa Biosystems, Wilmington, MA, USA) and the Applied Biosystems 7500/7500 Fast Real-Time PCR System (Thermo Fisher Scientific, Waltham, MA, USA), according to the manufacturer's instructions.

The cycling program included preliminary denaturation at $95{ }^{\circ} \mathrm{C}$ for $20 \mathrm{~s}$, followed by 40 cycles of denaturation at $95{ }^{\circ} \mathrm{C}$ for $3 \mathrm{~s}$, and annealing and elongation at $60{ }^{\circ} \mathrm{C}$ for $3 \mathrm{~s}$. Melting curve analysis confirmed that the PCR product did not contain non-specific by-products. TATA-binding protein (Tbp) was used as the internal normalizing control for the mRNA. The cycle threshold $(\mathrm{Ct})$ value of the target gene mRNAs was normalized to the $\mathrm{Ct}$ value of $\mathrm{Tbp}$ mRNA ( $2^{-\Delta \Delta \mathrm{Ct}}$ method) for all groups. The primer sequences used in this study are shown in Table 1.

\section{Statistical analysis}

Data are shown as mean \pm standard deviation (S.D.). For all measurements, two-way analysis of variance was used to evaluate the significance. In the case of significant $F$ values, comparisons were performed using Tukey's post-hoc test. Statistical analyses were conducted using GraphPad Prism 7 software (GraphPad, Inc., La Jolla, CA, USA). P values below 0.05 were considered significant.

\section{RESULTS}

\section{Body weight and weight of tissues}

The weight of the body and tissues of the mice are shown in Fig. 2. The final weight of the body and WAT showed a significant interaction among all groups. The HFD-Tr group had a significantly lower weight compared to the HFD-Sed group, although the HFD groups had higher weights compared to the ND groups.

The weight of the skeletal muscles showed a significant interaction among all groups. The HFD-Tr group had a significantly higher muscle weight compared to the HFD-Sed group, except for the soleus muscle. However, the HFD groups had lower muscle weights than the ND groups.

Table 1. Primer sequences used in real time - qRT - PCR analyses

\begin{tabular}{lcc}
\hline Gene name & \multicolumn{1}{c}{ Forward } & Reverse \\
\hline Tbp & CAGCCAAGATTCACGGTAGAT & CCAATGACTCCTATGACCCCTA \\
Renalase & GGGTGGGGATATAGGGGGAAG & GGTGATGTACTGAGCTCCCA \\
Comt & AGACCGCTACCTTCCAGACA & GTTCCCGGGACAATGACA \\
Mao- $a$ & CGGATATTCTCAGTCACCAATG & ATTTGGCCAGAGCCACCTA \\
Mao $b$ & CCAGAATCATCTCAACAACCAA & TCACTTGACCAGATCCACCA \\
\hline
\end{tabular}

Tbp, TATA-binding protein; Comt, catechol-O-methyltransferase; Mao-a, monoamine oxidase A; Mao-b, monoamine oxidase $\mathrm{B}$. 
Interaction: $p<0.01$

Exercise: $p<0.01$

Diet: $p<0.01$

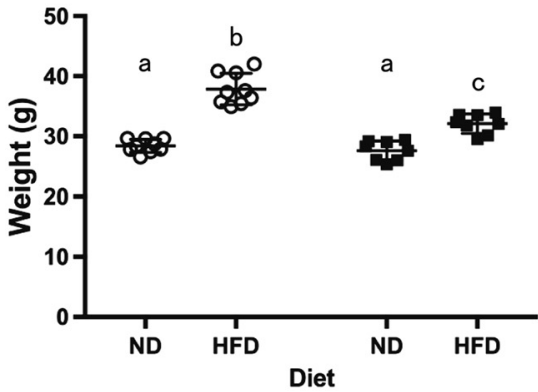

Interaction: $p<0.01$

Exercise: $p<0.01$

Diet: $p<0.01$
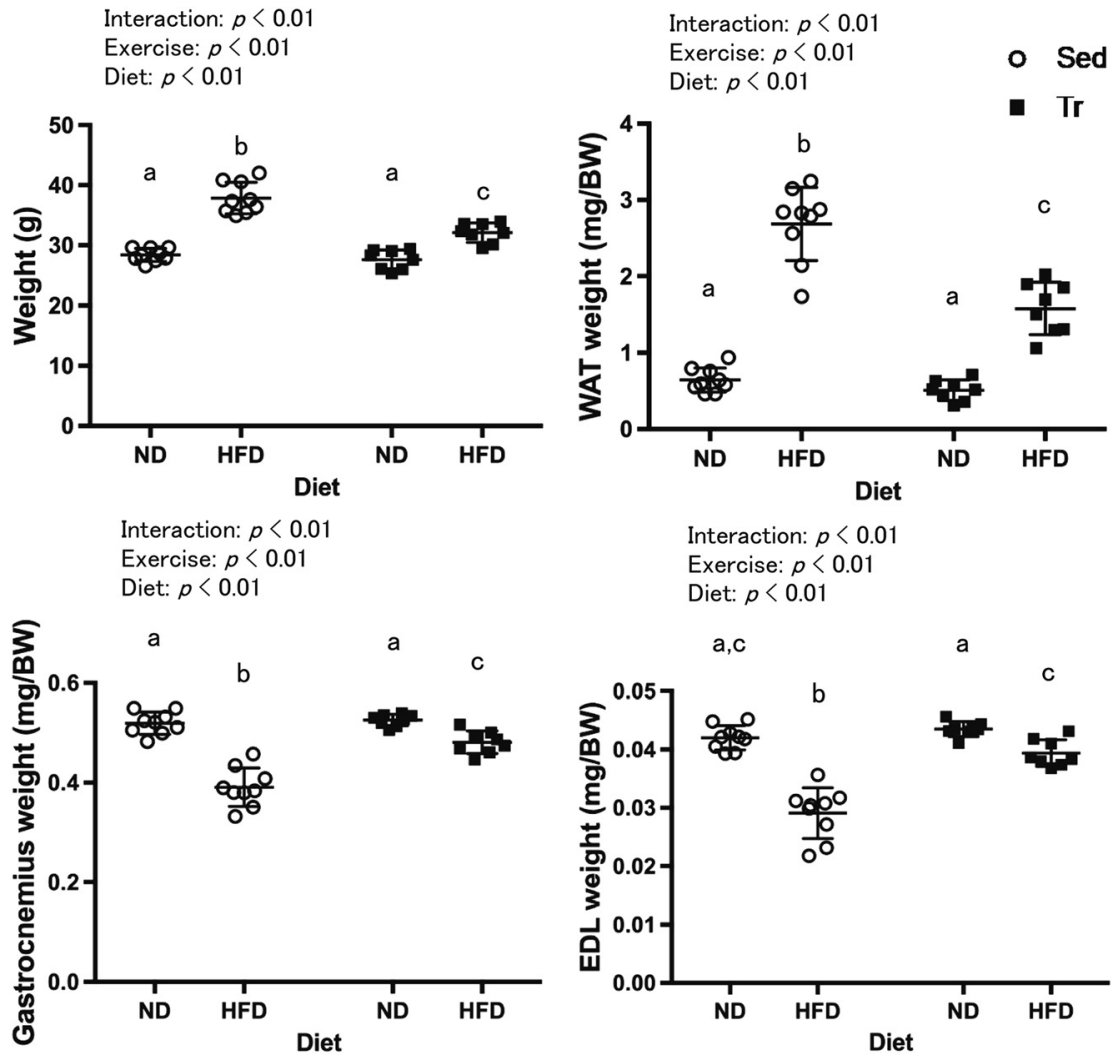

Interaction: $p<0.01$

Exercise: $p<0.01$

Diet: $p<0.01$

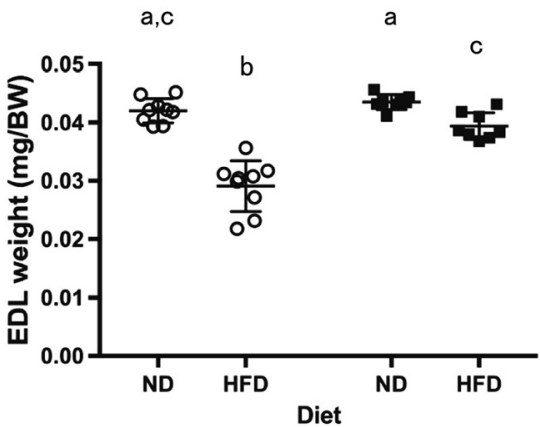

Interaction: $p<0.01$

Exercise: $p<0.01$

Diet: $p<0.01$

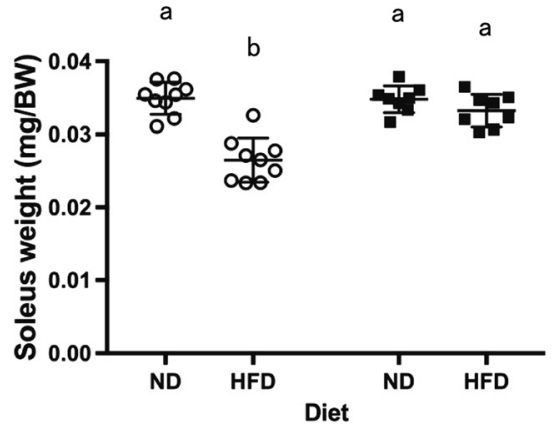

Fig. 2. Body weight and weight of WAT and skeletal muscles in mice fed a high-fat diet and subjected to exercise training.

Different characters indicate statistical significance $(P<0.05)$. ND, normal diet; HFD, high-fat diet; Sed, sedentary; $\mathrm{Tr}$, high-intensity exercise training; EDL, extensor digitorum longus; WAT, white adipose tissue; $n=9$ in Sed group; $n=8$ in high-intensity exercise training group 


\section{Blood lactate, plasma total cholesterol and glucose tolerance tests}

Blood lactate in the Tr groups was significantly higher than that in the Sed groups (Table 2). The plasma total cholesterol level in the HFD groups was higher than that in the ND groups (Table 2).

The results of the glucose tolerance test (GTT) showed a significant interaction among all groups (Fig. 3). Fasting blood glucose level was higher in the HFD groups than in the ND groups at $0 \mathrm{~min}$. The ND-Tr group showed a lower blood glucose level compared to the other groups at $15 \mathrm{~min}$. At 30 and $60 \mathrm{~min}$, there was a significant difference between ND and HFD groups. The HFD-Sed group showed a higher blood glucose level compared to the other groups at $120 \mathrm{~min}$.

\section{Renalase mRNA content in each tissue}

The change in renalase mRNA content in various tissues is shown in Fig. 4. Renalase mRNA content in the gastrocnemius and extensor digitorum longus (EDL) muscles in the Tr groups was higher than that in the Sed groups, whereas there was a significant interaction between

Table 2. Blood lactate and plasma total cholesterol

\begin{tabular}{lcc}
\hline Group & Lactate & total cholesterol \\
\hline ND-Sed & $2.0 \pm 0.4$ & $93.8 \pm 3.2$ \\
HFD-Sed & $1.6 \pm 0.2$ & $154.0 \pm 24.6^{\dagger \dagger}$ \\
ND-Tr & $3.1 \pm 0.8^{* *}$ & $87.3 \pm 6.1$ \\
HFD-Tr & $3.5 \pm 1.5^{* *}$ & $140.9 \pm 25.0^{\dagger \dagger}$ \\
\hline
\end{tabular}

Data showed as mean \pm SD. ${ }^{* *}$ and ${ }^{\dagger \dagger}$ indicated significant main effects $(P<0.01)$ vs. training or high fat diet, respectively. ND, normal diet; HFD, high fat diet; Sed, sedentary; Tr, high intensity exercise training.
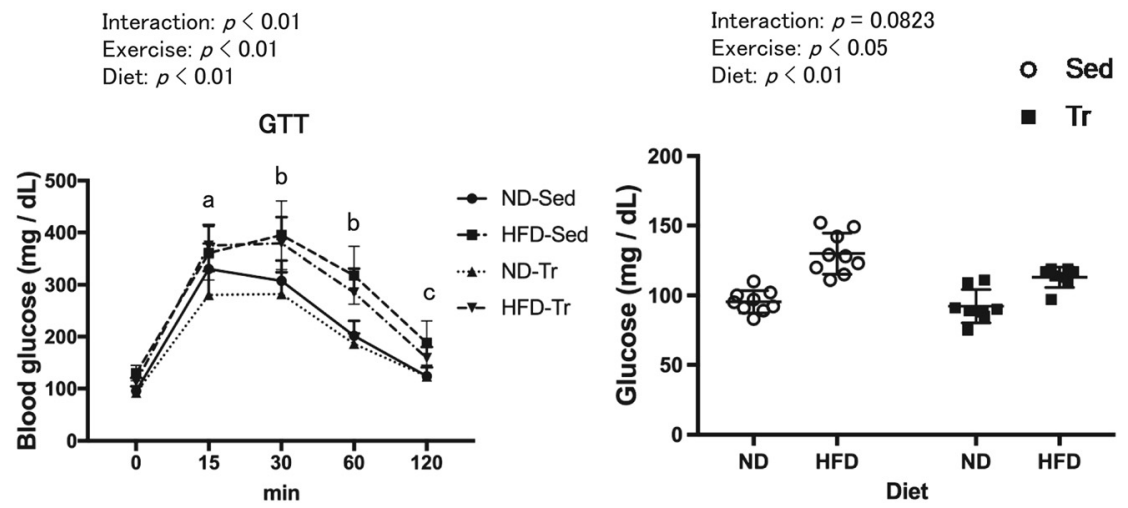

Fig. 3. Results of glucose tolerance test and fasting blood glucose of mice fed a high-fat diet and subjected to exercise training.

Characters indicates statistical significance $(P<0.05)$. a, ND-Tr group versus the other groups; $b$, ND groups versus HFD groups; c, HFD-Sed group versus ND groups. ND, normal diet; HFD, high-fat diet; Sed, sedentary;

Tr, high-intensity exercise training; $n=9$ in Sed group; $n=8$ in high-intensity exercise training group 


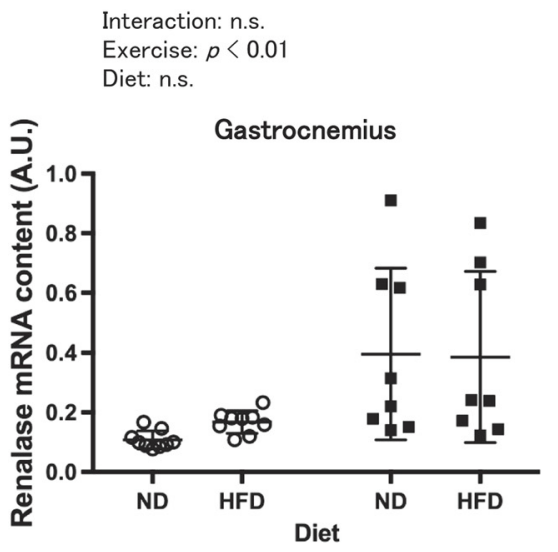

Interaction: $p<0.01$

Exercise: n.s.

Diet: $p<0.05$

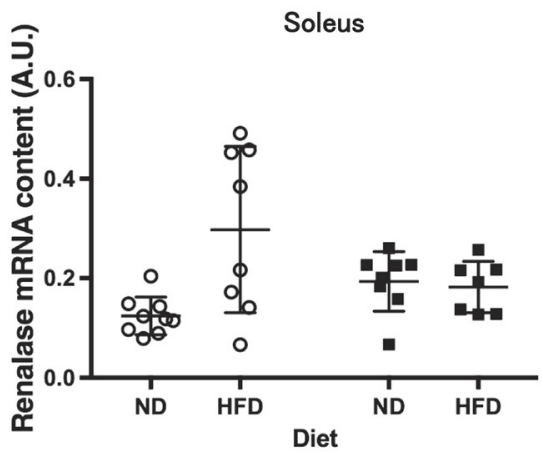

Interaction: n.s.

Exercise: $p<0.05 \quad$ o $\quad$ Sed

Diet: n.s.

- $\mathrm{Tr}$

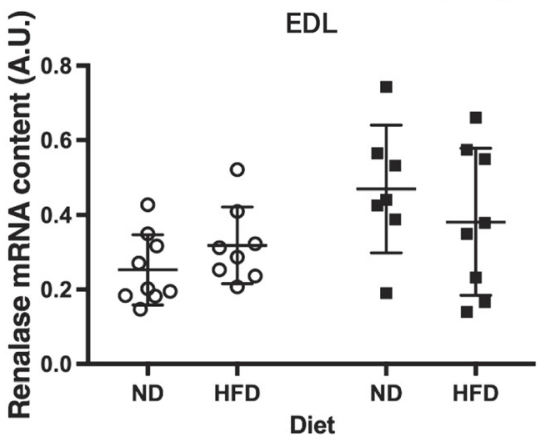

Interaction: $p<0.05$

Exercise: $p<0.01$

Diet: $p<0.01$

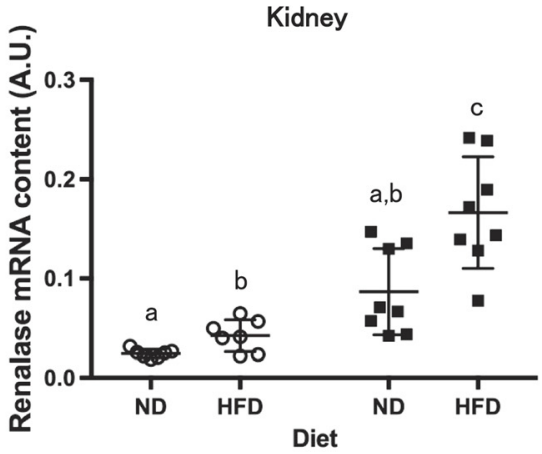

Fig. 4. Renalase mRNA expression in the skeletal muscles and kidneys in mice fed a high-fat diet and subjected to exercise training.

Different characters indicate statistical significance $(P<0.05)$. ND, normal diet; HFD, high-fat diet; Sed, sedentary; $\mathrm{Tr}$, high-intensity exercise training; EDL, extensor digitorum longus; $n=7-9$ in each group

exercise training and HFD in the soleus muscle. In addition, there was a significant interaction between exercise training and HFD in the kidneys.

\section{mRNA content of regulators related to metabolism of catecholamines}

Comt, Mao- $a$, and Mao- $b$ mRNA contents in various skeletal muscles are shown in Fig. 5. The amount of these mRNAs in the gastrocnemius and EDL muscles did not change in any of the groups. Mao-a and Mao-b mRNA contents in the soleus muscle did not change in any of the groups. However, Comt mRNA content was significantly higher in the soleus muscle. 

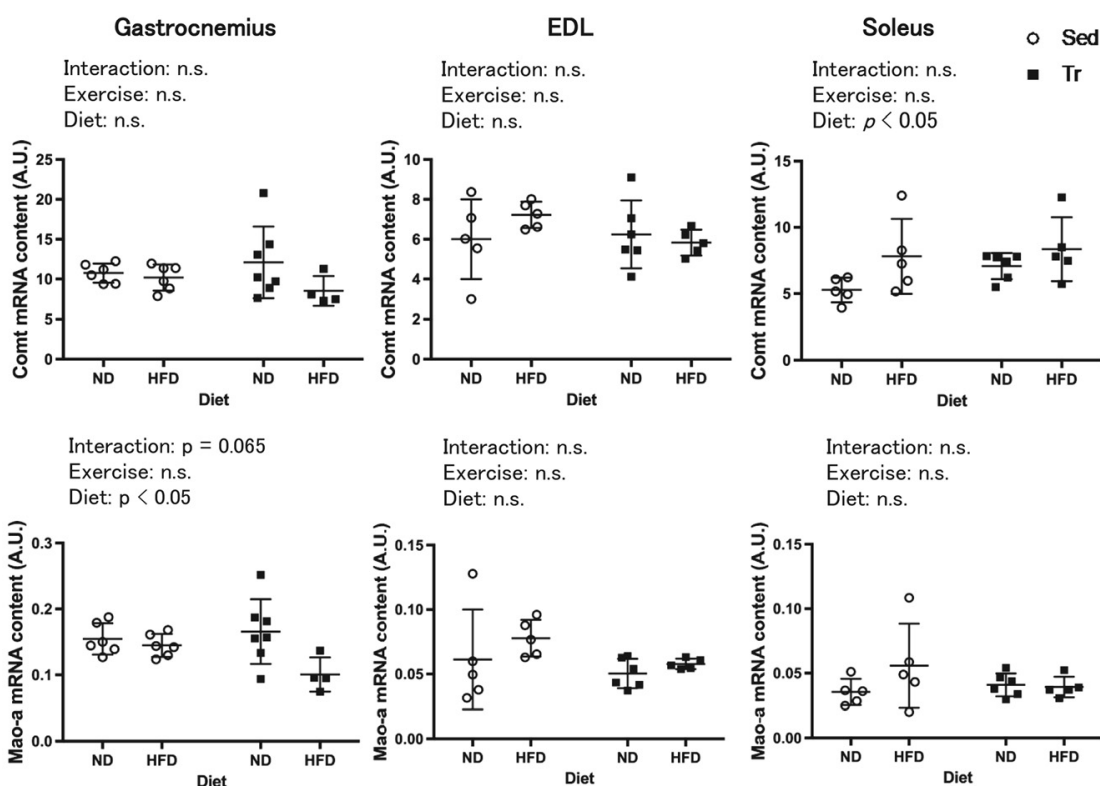

Interaction: n.s.

Exercise: n.s.

Diet: n.s.

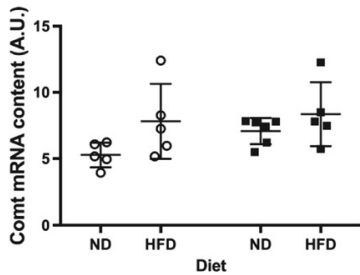

Interaction: n.s.

Exercise: n.s.

Diet: n.s.
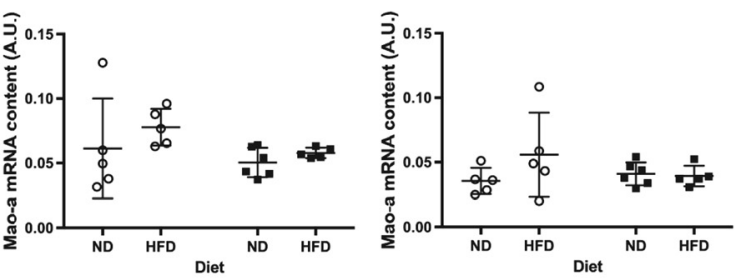

Interaction: $n$.s.

Exercise: n.s.

Diet: n.s.

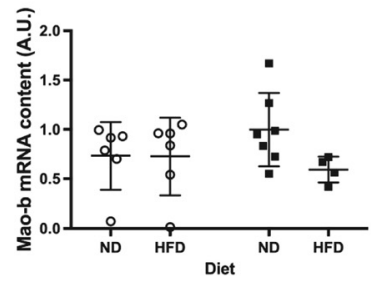

Interaction: n.s

Exercise: n.s.

Diet: n.s.

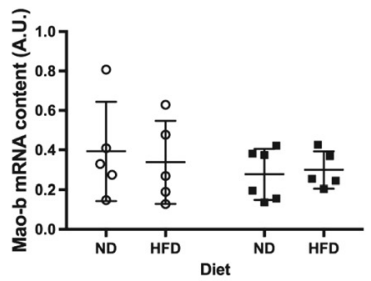

Interaction: n.s.

Exercise: n.s.

Diet: n.s.

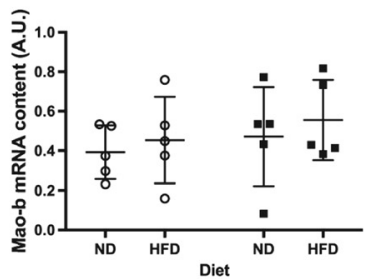

Fig. 5. COMT, MAO-A, and MAO-B mRNA content in skeletal muscles in mice fed a high-fat diet and subjected to exercise training.

ND, normal diet; HFD, high-fat diet; Sed, sedentary; Tr, high-intensity exercise training; EDL, extensor digitorum longus; COMT, catechol-O-methyltransferase; MAO-A, monoamine oxidase A; MAO-B, monoamine oxidase $\mathrm{B} ; n=4-6$ in each group

\section{DISCUSSION}

This study was performed to investigate the change in renalase mRNA content with highintensity exercise training and/or HFD for 8 weeks in mice. There was a significant difference in body weight and the weight of WAT between the HFD-Sed and HFD-Tr groups. The GTT results revealed that only the HFD-Sed group had significantly higher blood glucose than the ND groups at $120 \mathrm{~min}$. Thus, high-intensity exercise training for 8 weeks improved obesity induced by HFD in this study. Okamoto et al. reported the lactate threshold with moderate-intensity exercise in mice at the speed of $17.5-20 \mathrm{~m} / \mathrm{min}$. In this study, exercise 
intensity was set to $25 \mathrm{~m} / \mathrm{min}$, which is estimated to be high intensity for mice [16]. Moreover, the blood lactate in the Tr groups was higher than that in the Sed groups. This demonstrates that the mice in the Tr groups completed the training protocol, high intensity exercise. Therefore, in this study, the obesity experimental model and exercise training were reliable.

The $\operatorname{Tr}$ groups had increased renalase mRNA content in the gastrocnemius and EDL muscles compared to the Sed groups. It has been reported that exercise training results in a decrease in blood catecholamine levels [1]. Renalase secreted from the kidneys is known to metabolize circulating catecholamines in the blood $[7,8]$. Therefore, our results suggest that renalase in the skeletal muscle also metabolizes circulating catecholamines. While exercise training did not lead to a change in renalase mRNA content in the soleus muscle, the HFDSed group had higher renalase mRNA content than the ND-Sed group for 8 weeks. Highintensity exercise mainly recruits fast-twitch fibers in the EDL muscle [17]. CzarkowskaPaczek et al. [18] showed that renalase expression increased in the white fibers of the gastrocnemius muscle; however, there was no change in the red fibers immediately after exercise. Tokinoya et al. [15] reported that the increase in renalase expression in the skeletal muscles is related to exercise intensity and muscle fiber type. The effect of low-intensity exercise training on the renalase mRNA content in the soleus muscle should be explored further. Therefore, renalase mRNA content in the soleus muscle might be changed by low intensity exercise training.

Interestingly, the renalase mRNA content in the kidneys was increased by both HFD and exercise training despite being increased in the skeletal muscle. Type 2 diabetes (T2DM) and acute exercise induce an increase in circulating catecholamines $[1,19]$. Both obesity and exercise are related to hypertension $[20,21]$. In previous studies, renalase concentration in T2DM showed a significantly higher value than that in healthy subjects [1]. It is likely that this is brought about by increased renal secretion, because our findings showed that renalase mRNA content in the kidney only increased in the HFD group. On the other hand, it is unclear why renalase mRNA content in the kidney is increased by exercise training. Renalase is classified as a novel monoamine oxidase in addition to MAO-A and -B $[9,14]$. It has been reported that exercise training results in a decrease in blood catecholamine levels [1]. Therefore, it might be possible that renalase in the kidney was related to a new mechanism underlining this phenomenon.

Our results regarding COMT, MAO-A, and $-\mathrm{B}$, which are involved in catecholamine metabolism, showed that the level of the mRNAs of these enzymes did not change with HFD and exercise training except in the soleus muscle. In previous studies, increased renalase concentration in the blood has been shown to be positively correlated with catecholamines in the blood in some patients $[1,11]$. Moreover, inhibitors of COMT and MAO-A do not have any effect on blood parameters and plasma catecholamines during exercise [6]. Therefore, renalase in the skeletal muscle and kidney metabolizes circulating catecholamines during exercise training. In addition, in this study renalase in the blood may have been increased by exercise training instead of COMT, MAO-A, and MAO-B to metabolize the circulating catecholamines.

In summary, renalase mRNA content may have increased to metabolize catecholamines, as the mRNA of COMT, MAO-A, and MAO-B remained unchanged in the skeletal muscles with exercise training and obesity. Thus, our study demonstrated that renalase can lead to lipolysis via catecholamines and ameliorate obesity. 
Conflict of Interest statement: The authors declare that there are no conflicts of interest.

\section{ACKNOWLEDGMENTS}

We appreciated the kindness of all animals. We are grateful to Dr. Yasuko Yoshida and Ms. Nanami Sekine for their assistance in the discussion of this study. We would like to thank Editage (www.editage.com) for English language editing. This work was supported by the JSPS KAKENHI Grant Number 18J10187.

\section{ABBREVIATIONS}

COMT catechol-O-methyltransferase

MAO-A monoamine oxidase A

MAO-B monoamine oxidase $\mathrm{B}$

\section{REFERENCES}

1. Wang F, Huang B, Li J, Liu L, Wang N. Renalase might be associated with hypertension and insulin resistance in Type 2 diabetes. Ren Fail 2014; 36: 552-6.

2. De Oliveira Sá G, dos Santos Neves V, de Oliveira Fraga SR, Souza-Mello V, Barbosa-da-Silva S. High-intensity interval training has beneficial effects on cardiac remodeling through local renin-angiotensin system modulation in mice fed high-fat or high-fructose diets. Life Sci 2017; 189: 8-17.

3. Hafstad AD, Lund J, Hadler-Olsen E, Höper AC, Larsen TS, Aasum E. High- and moderate-Intensity training normalizes ventricular function and mechanoenergetics in mice with diet-Induced obesity. Diabetes 2013; 62: 2287-94.

4. Rogers PJ, Tyce GM, Weinshilboum RM, O’Connor DT, Bailey KR, Bove AA. Catecholamine metabolic pathways and exercise training: plasma and urine catecholamines, metabolic enzymes, and chromogranin-A. Circulation 1991; 84: 2346-56.

5. Winder WW, Hagberg JM, Hickson RC, Ehsani AA, McLane JA. Time course of sympathoadrenal adaptation to endurance exercise training in man. J Appl Physiol Respir Environ Exerc Physiol 1987; 45: 370-4.

6. Illi A, Sundberg S, Ojala-Karlsson P, Scheinin M, Gordin A. Simultaneous inhibition of catechol-O-methyltransferase and monoamine oxidase A: effects on hemodynamics and catecholamine metabolism in healthy volunteers. Clin Pharmacol Ther 1996; 59: 450-7.

7. Desir GV, Tang L, Wang P, Li G, Sampaio-Maia B, Quelhas-Santos J, et al. Renalase lowers ambulatory blood pressure by metabolizing circulating adrenaline. J Am Heart Assoc 2012.

8. Wu Y, Xu J, Velazquez H, Wang P, Li G, Liu D, et al. Renalase deficiency aggravates ischemic myocardial damage. Kidney Int 2011; 79: 853-60.

9. Xu J, Li G, Wang P, Velazquez H, Yao X, Li Y, et al. Renalase is a novel, soluble monoamine oxidase that regulates cardiac function and blood pressure. J Clin Invest 2005; 115: 1275-80.

10. Yoshida Y, Sugasawa T, Hoshino M, Tokinoya K, Ishikura K, Ohmori H, et al. Transient changes in serum renalase concentration during long-distance running: the case of an amateur runner under continuous training. J Phys Fit Sport Med 2017; 6: 159-66. 
11. Zbroch E, Musialowska D, Koc-Zorawska E, Malyszko J. Age influence on renalase and catecholamines concentration in hypertensive patients, including maintained dialysis. Clin Interv Aging 2016; 11: 1545-50.

12. Tokinoya K, Yoshida Y, Sugasawa T, Takekoshi K. Moderate-intensity exercise increases renalase levels in the blood and skeletal muscle of rats. FEBS Open Bio 2020; 1-8.

13. Yoshida Y, Sugasawa T, Tokinoya K, Namba S, Takekoshi K. Epinephrine upregulates renalase expression in cultured C2C12 muscle cells. Int J Anal Bio-Sci. 2017; 5: 61-5.

14. Sonawane PJ, Gupta V, Sasi BK, Kalyani A, Natarajan B, Khan AA, et al. Transcriptional regulation of the novel monoamine oxidase renalase: crucial roles of transcription factors Sp1, STAT3, and ZBP89. Biochemistry 2014; 53(44): 6878-92.

15. Tokinoya K, Shiromoto J, Sugasawa T, Yoshida Y, Aoki K, Nakagawa Y, et al. Influence of acute exercise on renalase and its regulatory mechanism. Life Sci 2018; 210: 235-42.

16. Okamoto M, Yamamura Y, Liu Y-F, Min-Chul L, Matsui T, Shima T, et al. Hormetic effects by exercise on hippocampal neurogenesis with glucocorticoid signaling. Brain Plast 2015; 1: 149-58.

17. Sieck GC, Fournier M. Diaphragm motor unit recruitment during ventilatory and nonventilatory behaviors. J Appl Physiol 1989; 66: 2539-45.

18. Czarkowska-Paczek B, Zendzian-Piotrowska M, Gala K, Sobol M, Paczek L. Exercise differentially regulates renalase expression in skeletal muscle and kidney. Tohoku J Exp Med 2013; 231: 321-9.

19. Zouhal H, Jacob C, Delamarche P, Gratas-Delamarche A. Catecholamines and the effects of exercise, training and gender. Sport Med 2008; 38: 401-23.

20. Fantin F, Giani A, Zoico E, Rossi AP, Mazzali G, Zamboni M. Weight loss and hypertension in obese subjects. Nutrients 2019.

21. Katch VL, McArdle WD, Katch FI. Essentials of exercise physiology. In: The cardiovascular System and exercise. 4th ed.; 2011. p. 306-11. 